\title{
Agent-based distributed performance measurement system for ITSP projects
}

\begin{abstract}
Measuring the development of an enterprise software project progress and performance is crucial to assure a project follow its path. This attention stems in parts from the frequent reports of high profile cases of mismanaged in IT projects particularly in enterprise level such as information technology strategic planning (ITSP) projects. Many project performance measurement models and frameworks have been proposed to address the problem, however, they are usually hard to manage and inefficient in practice due to the complexity, distribution and dynamism of these types of projects. A large and growing body of literature has shown the advantages of employing the agent technology in distributed, dynamic, and complex environments. Therefore, in this study the advantages of the agent technology will be applied to improve the progress and measurement process of the software project performance measurement approaches. In this paper, a multi-agent system architectural model with the focus in implementation phase of the ITSP projects is proposed to promote and facilitate the process of project performance measurement. Furthermore the prototype of the proposed solution is explained and the evaluation approach is discussed.
\end{abstract}

Keyword: ITSP; Multi-agent systems; Software project progress; Performance measurement 